\title{
Characteristics of dental pulp in human upper first premolar teeth based on immunohistochemical and morphometric examinations
}

\author{
Joanna Maria Tomaszewska1, Bogdan Miskowiak ${ }^{2,3}$, Teresa Matthews-Brzozowska ${ }^{1}$, \\ Piotr Wierzbicki ${ }^{4}$
}

${ }^{1}$ Department of Maxillofacial Orthopaedics and Orthodontics, Poznan University

of Medical Sciences, Poland

${ }^{2}$ Department of Optometry and Biology of Visual System, Poznan University of Medical Sciences, Poland

${ }^{3}$ Department of Histology and Embryology, Poznan University of Medical Sciences, Poland

${ }^{4}$ Department of Histology, Medical University of Gdansk, Poland

\begin{abstract}
Teeth extracted for orthodontic reasons are commonly considered as healthy. Therefore, it is possible to examine structure of the dental pulp can be fully recognized and how it is affected by malocclusion. The aim of the study was to evaluate by immunohistochemistry (IHC) and morphometry dental pulp in human upper first premolar teeth extracted for orthodontic reasons. The material comprised 36 teeth of 20 patients in the age range 16-26 years. By the use of IHC markers the presence of immunocompetent cells (CD20, CD45RO, and CD68), blood vessels (CD31) and nerves (PGP9.5) were examined in the pulp. Inflammatory infiltrates and tissue atrophy were observed in 24 and 10 teeth, respectively. Strong positive correlation between the width of the odontoblastic layer, the number of rows of odontoblast nuclei and the increase of MVA (microvessel area) in the pulp of atrophic teeth was found. The cellular infiltrations found in $\mathrm{H} \& \mathrm{E}$-stained sections were identified by IHC as memory T cells $(\mathrm{CD} 45 \mathrm{RO}+)$ and $\mathrm{B}$ lymphocytes $(\mathrm{CD} 20+)$ with macrophages $(\mathrm{CD} 68+)$ present at the periphery. The CD20 antigen was intensively expressed in 13 teeth, CD45RO in 33 teeth, and CD68 in 20 teeth. Thus, despite the lack of any clinical signs of pulp disease many teeth extracted for orthodontic reasons show focal pulp inflammation and atrophy which probably results from the malocclusion stress accompanying teeth crowding. (Folia Histochemica et Cytobiologica 2013, Vol. 51, No. 2, 149-155)
\end{abstract}

Key words: dental pulp, premolars, orthodontics, morphometry, immunohistochemistry, CD20, CD45RO, CD68

\section{Introduction}

Dental pulp is a loose connective tissue of ectomesenchymal origin, resembling immature gelatinous tissue, and is enclosed in a rigid framework made of

Correspondence address: J. Tomaszewska

Department of Maxillofacial Orthopaedics and Orthodontics; Bukowska St. 70, 60-812 Poznan, Poland; tel.: +48609047 339; fax: +48618547068;

e-mail: j.tomaszewska@interia.eu hard dental structures [1]. In the direction from dentin to tooth centre, pulp has four microscopic zones: odontoblast layer, cell-poor zone, cell-rich zone and pulp proper [2].

The odontoblast layer is actually composed of the cell bodies of these cells since their processes pass through dentinal tubules and the cell-poor zone [3] and the multilayer illusion is the result of odontoblast nuclei positioned on different levels. The cell-poor zone, as the name indicates, is relatively free of cells, whereas the cell-rich zone contains a higher propor- 
tion of cellular elements than the rest of the tissue. Pulp proper composes the central mass of tissue and resembles other connective tissues; it is here where the large blood vessels and nerves are present. Dental pulp is a highly vascularized tissue with many small blood vessels comprising arterioles, capillaries and venules [4]. Dental pulp is also highly innervated autonomic and sensory fibres [5]. The condition of dental pulp is very dependent on the state of the dentin or enamel; any injury to them affects the pulp. Depending on type of irritant and its potency, this can lead to either the degeneration of pulp tissue or to smaller changes which can be repaired due to the regenerative capacity of the pulp. Pulp inflammation is a dynamic and complex process involving a number of neural, vascular and immunological reactions $[5,6]$, often varied in different tissue regions [7]. In the case of severe chronic inflammation the tissue becomes necrotic, whereas chronic irritation of smaller intensity can lead to pulp degeneration and atrophy [8].

Many studies of the pulp tissue concerned influence of orthodontic treatment, including effects of applied forces being checked both during and after treatment $[9,10]$. However, most of the investigators did not take into account the possibility that some changes can occur even before treatment as a result of the malocclusion stress which accompanies crowding. It is crowding as well as bialveolar protrusion that are the most common reasons for the extractions of premolars, usually the first ones [11, 12]. Although in some studies the morphology of the dental pulp of upper first premolar teeth had been investigated, there was no attempt to correlate the histological findings with the orthodontic findings and their possible correlations.

The aim of this study was to evaluate the histological structure of the dental pulp of human upper first premolar teeth extracted for orthodontic reasons and correlate these observations with the presence of immune cells, vessels and nerve fibres of the pulp identified by immunohistochemistry (IHC). The secondary goal was to estimate if the occurrence of morphological changes in the pulp structure can have an influence on planning orthodontic treatment.

\section{Material and methods}

The research material consisted of 36 human first upper premolar teeth (18 left and 18 right) extracted for orthodontic reasons from 20 patients 16-26 years old. Extractions were performed because of crowding manifested by a lack of space for canines. All teeth used in the research were fully erupted, without any signs of periodontal disease or caries. The research project obtained ethical approval from the Bioethical Committee of Poznan University of Medical Sciences (138/07).
Immediately after extraction, the teeth were fixed in $10 \%$ phosphate-buffered formalin ( $\mathrm{pH} 7,0$ ) for 7 days. Then they were sectioned with a diamond bur along the long axis on lingual and buccal surfaces and split in halves [13]. The pulp was carefully removed from hard structures with dental tweezers, dehydrated in alcohol and embedded in paraffin. Paraffin blocks were cut with microtome to $5 \mu \mathrm{m}$ thick sections and mounted on glass slides (SuperFrost ${ }^{\circledR}$ ). Depending on the amount of material obtained from each tooth 4 to 12 slides were stained with hematoxylin and eosin (H\&E) and 15 to 25 slides were submitted for immunohistochemical examination using the biotin-streptavidin system and tyramine signal amplification (the immunomax technique). The following antigens were identified by the use of mouse monoclonal anti-human antibodies: CD20, B-cells marker (clone L26, Novus Biologicals, Littleton, CO, USA, 1:200), CD45RO, memory T cells marker (clone UCHL1, Novus Biologicals, 1:5000), CD68, monocytes/ /macrophages marker (clone PG-M1, Novus Biologicals, 1:200), blood vessels CD31, endothelial cell marker (clone JC70A, DakoCytomation, Golstrup, Denmark, 1:200), and nerve fibres PGP9.5, general nerve cell marker (clone UCH -L1, Santa Cruz Biotechnology, Heidelberg, Germany, 1:100). After incubating sections with primary antibody at $21^{\circ} \mathrm{C}$, the secondary antibody (biotinylated goat anti-mouse sc-2072; Santa Cruz Biotechnology) was applied and the slides were incubated with streptavidin-peroxidase complex (Vectastain Elite; Vector Laboratories, Burlingame, CA, USA). Localization of the reaction was visualized by the use of diaminobenzidine (DAB) and cellular nuclei were counterstained with hematoxilin.

The pulp specimens obtained were examined under a Nikon Eclipse E600 light microscope with a CCTV colour camera (Panasonic WV-CP242) coupled to MicroImage v. 4.0 image analyser (Media Cybernetics). For analysis 20 images from each tooth were chosen providing on the whole 720 microscopic images.

In the qualitative evaluation of the pulp condition the following parameters were considered: cytological pattern of all pulp zones, vasculature, innervation, occurrence of cellular infiltrations and tissue atrophy. For the morphometric measurements the researcher identified and counted the histological structures and cells seen at a magnification of $\times 100$ and in an observed area of $0.25 \mathrm{~mm}^{2}$. For quantitative evaluations the number of immunopositive cells for each marker was counted, the width of the odontoblastic layer was measured and diameter, number of cross-sections and relative area of blood vessel (as percentage of the total measured area) was determined. Statistical analysis was performed using the program Statistica v. 7.1 (Statsoft Polska, Cracow, Poland). The Mann-Whitney U test was used and the Spearman correlation coefficient was calculated, with $\mathrm{p}<0.05$ being considered statistically significant. 


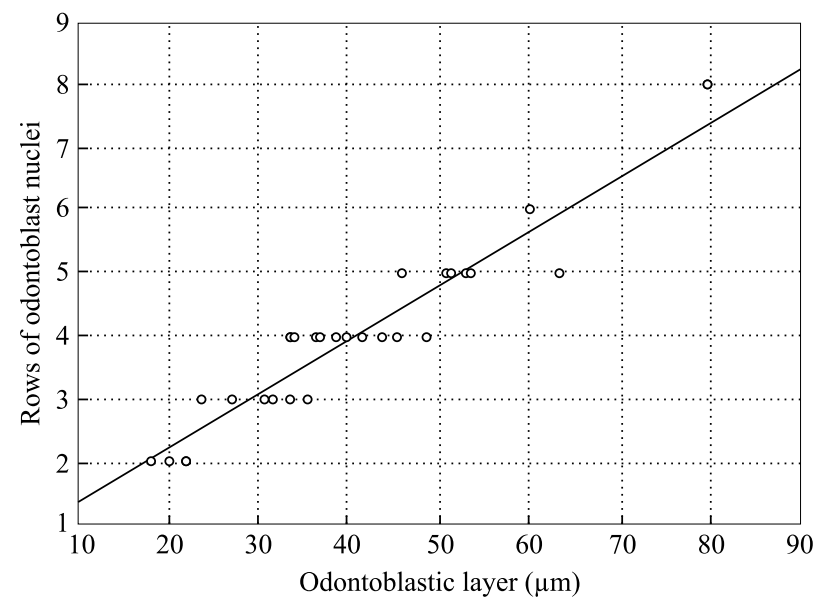

Figure 1. Correlation between the width of the odontoblastic layer and the number of rows of odontoblasts' nuclei (in 36 teeth)

\section{Results}

\section{Histoarchitecture of dental pulp}

The odontoblast layer was characterized by a variability in its width depending on localization, it was thinner in the pulp horns and also a lack of continuity in the layer was observed. Morphometric analysis showed that the width of the odontoblastic layer was $40.0 \pm$ $\pm 13.9 \mu \mathrm{m}$ (mean $\pm \mathrm{SD})$, and the mean number of rows of odontoblast nuclei was $4 \pm 1$. Between these two parameters a strong positive correlation $(\mathrm{r}=0.95)$ was found, the number of rows of odontoblast nuclei grew by a factor of 1 when the width of the layer increased by $10 \mu \mathrm{m}$ (Figure 1 ).

The structure of the dental pulp of teeth without dental atrophy and inflammatory cell infiltrates were typical as described by other authors $[10,14]$. The cell -poor zone was very variable, from distinct without any cellular elements to indistinct one showing continuity with the cell-rich zone. These kinds of differences were seen not only between particular teeth but also between sections of the same tooth and even on the same slide. The cell-rich zone was clearly visible with some minor differences in respect to the cell size and cell density. The central pulp comprised the majority of the pulp, with the pattern of vasculature and innervation being clearly visible in the zone.

\section{Dental pulp vasculature}

Arterioles with reduced muscular layer branched into capillary network under the odontoblast layer. In the cell-poor zone capillaries often made loops and crossed over each other whereas bigger vessels ran straight across the radicular and adjacent coronal pulp. In quantitative examination of the non-atrophic teeth the pulp vasculature turned out to vary significantly between specimens, which was indicated by large mean deviations in respect to vessel diameter, MVA (microvessel area) and MVD (microvessel density) (Table 1). Weak expression of the CD31 marker, considered as marker of endothelial cells, was observed in 34 teeth. Intensified expressions of CD31 were observed in the pulp horns (Figure 2A).

\section{Dental pulp innervation}

Nerve fibres with hardly any ramifications accompanied larger vessels making with them distinct bundles; under the cell-rich zone they branched into small bundles and single fibres creating a plexus below the odontoblast layer. Expression of

Table 1. Morphometric analysis of pulp vessels $(n=36)$

\begin{tabular}{|l|l|l|l|}
\hline & Diameter $[\boldsymbol{\mu m}]$ & MVD $\left(\mathbf{p e r} \mathbf{1} \mathbf{~ m m}^{2}\right)$ & MVA $(\boldsymbol{\%})$ \\
\hline Mean $\bar{\chi}$ & 27,6 & 42 & 12,2 \\
\hline Median $M$ & 27,6 & 37 & 10,3 \\
\hline Minimum $x_{\min }$ & 13,9 & 20 & 4,4 \\
\hline Maximum $x_{\max }$ & 51,6 & 110 & 26,8 \\
\hline Lower quartile $Q_{1}$ & 21,2 & 27 & 8,0 \\
\hline Upper quartile $Q_{3}$ & 34,5 & 45 & 14,9 \\
\hline Standard deviation $s$ & 8,1 & 21 & 5,6 \\
\hline Coefficient of variation $V_{x}$ & $29 \%$ & $50 \%$ & $46 \%$ \\
\hline
\end{tabular}




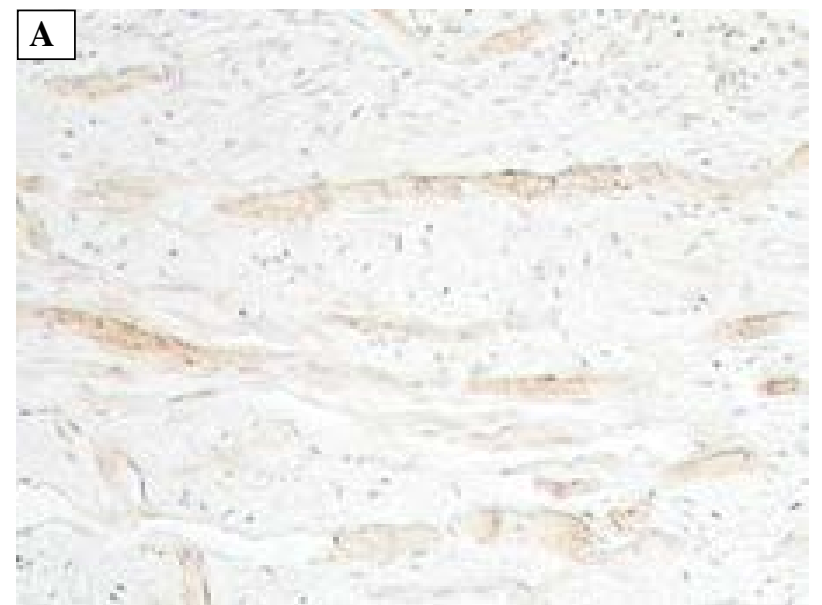

Figure 2. Expression of CD31 (A) in blood vessels, and PGP9.5 Original magnification $\times 100$

the PGP9.5 marker was found in 23 teeth mainly in medium-size and large nerve fibres localized in bundles in the centre of the radicular and coronal pulp and in the subodontoblastic plexus, however, not in thin axons passing through the odontoblast layer. Intense expression of PGP9.5 was observed in the pulp horns (Figure 2B).

\section{Dental pulp atrophy}

Tissue atrophy occurred in 10/36 teeth. This was characterized by the appearance of a network of small vessels with oval loops and a lack of cellular elements in relatively small areas (mean $0.15 \mathrm{~mm}^{2}$ ) surrounded by normal pulp tissue. Larger blood vessels were present in the normal tissue which surrounded the atrophic areas. Statistical analysis revealed significant differences between pulps with and without atrophy in respect to MVA, being $10.5 \%$ and $14.2 \%$, respectively.

\section{The assessment of inflammatory infiltrates}

Cellular infiltrates of various sizes were observed in 24 teeth. Infiltrations were usually localized in the subodontoblast zone, in the vicinity of the cell-rich zone or just below odontoblastic layer. The cells composing the bulk of this infiltrate showed typical lymphocyte morphology while the cells accompanying them, seen on the borders of infiltrates, showed typical various shapes of macrophages: round, oval, irregular, spindle, and dendritic (Figure 3A).

Expression of the CD20, B cell marker, was found in 13 teeth. CD20+ cells usually appeared in a diffuse pattern and in 3 teeth with dental pulp with inflammatory infiltrates they made clusters. Expression of the memory $\mathrm{T}$ cell marker, CD45RO, was found in

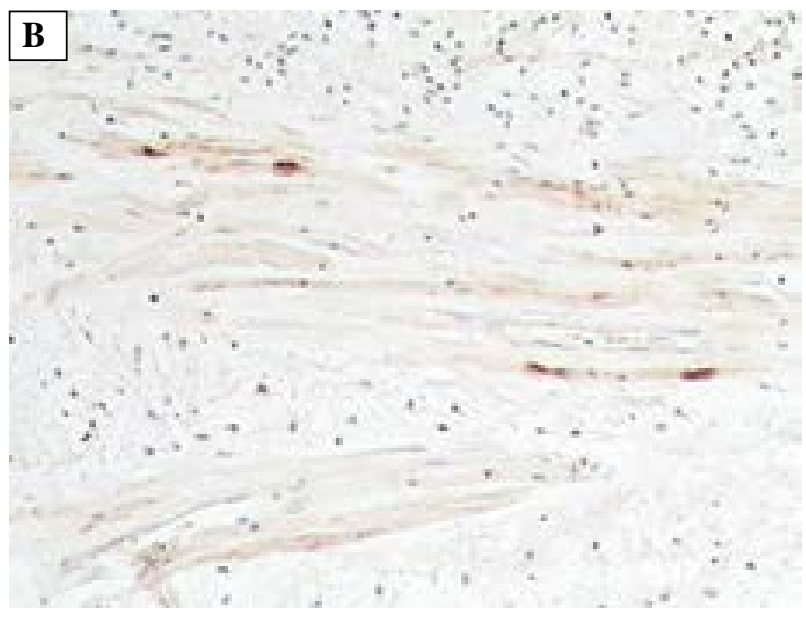

5 (B) in nerve fibres in the pulp horns as shown by IHC.

33 teeth. CD45RO + cells also usually appeared in a diffuse pattern, however, in teeth with inflammatory infiltrate in 5 cases they formed clusters. CD68+ cells were found in 20 teeth. They always showed a diffuse distribution pattern on the infiltrate boundaries and did not form clusters. Figure 3 shows the arrangement of inflammatory infiltrates and cells comprising them.

\section{Discussion}

Histological examination of specimens stained with $H \& E$ allowed a general qualitative evaluation of the pulp condition including observations of their morphological zones, the localization and arrangements of vessels and larger nerve fibre bundles, as well as quantitative changes in the cellular elements.

Examinations of the odontoblast layer confirmed data in the literature concerning the height of odontoblasts, $30-40 \mu \mathrm{m}$, and the number of rows of odontoblast nuclei, 2-8 [15]. Furthermore, the morphometric analysis allowed to find an original observation related to the correlation between the number of rows of odontoblasts' nuclei and the height of odontoblasts' layer. The often seen lack of continuity in the odontoblast layer most probably results from, as other authors have stated, the methods of sample collection; both teeth extractions and pulp removal from the hard tissues. However, it could also be a degeneration processes of the cells caused by pathogenic factors. Histological patterns in cell-poor and cell-rich zones turned out to be exceptionally variable in our research. The cell-poor zone was equally often observed and unseen, with the cell-rich zone being evident on every specimen but with some changes in the number of its cellular elements. The variable microarchitecture of these zones of dental 

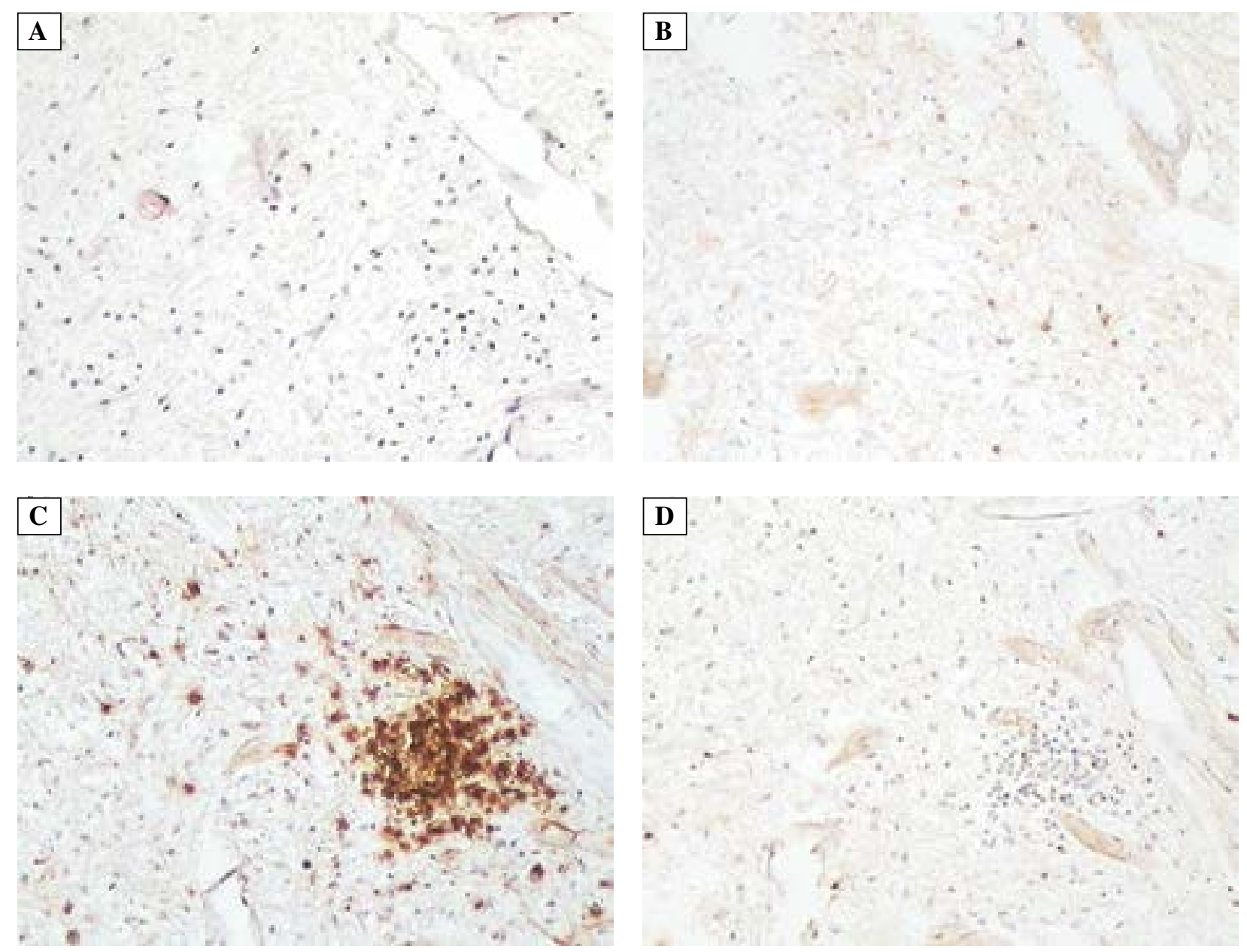

Figure 3. Serial sections of the pulp showing the same cellular infiltration by (A) H\&E staining, and immunocytochemical staining demonstrating (B) CD20-, (C) CD45RO- and (D) CD68-positive cells. $\times 100$

pulp proves the intense activity of the pulp. Large differences within one slide can prove that different activities take place in different areas of the pulp of a particular tooth, and can also prove the unequal exposure of that tooth to external factors.

Dental pulp is a blood-rich tissue, which is an effect of its high metabolic activity, and for this reason one of the most important determinants of pulp condition is its vasculature $[15,16]$.

The mean value of vessel diameter for all the teeth measured was between 21.2 and $34.5 \mu \mathrm{m}$ which is in agreement with the observations of other authors [16]. It is well known that bigger arteries enter the tooth via the apical foramina and immediately start giving off numerous branches thus reducing their own diameter [4].

The next parameter used was the number of vessel cross-sections per $1 \mathrm{~mm}^{2}$ of tissue, that is MVD (microvessel density). The results obtained in our study $\left(42 \pm 21\right.$ per $\left.1 \mathrm{~mm}^{2}\right)$ did not agree with other authors' results: $90 \pm 27.5$ in healthy tissue and $56.7 \pm 21.2$ in tissue with irreversible inflammation [17] along with $19.7 \pm 4.1$ in healthy and $25.3 \pm 7.2$ in inflamed pulp [1]. Although the methods of morphometric analysis were similar, there is a huge diversity in the results among various studies and therefore consideration should be given as to whether this method is adequate for the examination of human premolar pulp. The dental pulp is a relatively small tissue and there is not always the chance to obtain a proper cross-section. Interestingly, the estimates of relative vessel area [18] gave similar results between various investigations. We found that that blood vessels on average occupied $12.2 \%$ of the pulp; other authors reported similar vessel area in primary molars and a lower one in secondary teeth [4].

Other observations connected with pulp vasculature were the atrophic changes noticed in part of our research material. Atrophy is a decrease in the size and/or number of cellular elements in the dental pulp due to insufficient blood supply. Because of its resemblance to artifacts arising from wrong 
tissue preparation some authors do not recognize its occurrence. Examinations performed in this study confirmed that areas of atrophy could be found in the dental pulp [20]. It is well known that ischaemia is a local disturbance of the circulation, resulting in an insufficient blood supply. Pressure on the vessel wall by an inflammatory infiltrate is one of the causes of ischeamia, and a reduction of blood supply may lead to cell death. The consequences for this state depend mostly on its level and duration. These can be alleviated by the quick and effective application of collateral circulation, which could explain the fact that in our research material the vessels in pulp with atrophy occupied a larger tissue volume than in pulp without atrophic changes.

In classical histological examinations of the $\mathrm{H} \& \mathrm{E}$ stained specimens a limited local increase in the number of cellular elements were observed, which could be evidence of inflammatory infiltration. From other studies it is known that dental pulp contains large number of resident immunocompetent cells which become recruited in the case of pulp injury [14]. Thus, we found permanent presence of T-cells in the dental pulp, however, B lymphocytes identified by CD20 expression were observed in only 13/36 teeth. Because the teeth used in our research were exposed to chronic irritation due to the malocclusion, mononuclear cells were expected to form inflammatory infiltrations. We also stated the presence of macrophages (CD68+ cells) around those cellular infiltrations, what can be explained by their function which is antigen presentation and phagocytosis.

There are no obvious changes in innervation in the case of pulp inflammatory changes though it can be seen that the fibres around infiltration are more irregular and more ramified. When it comes to pulp tissue destruction the nerve fibres are still relatively intact showing only slight deviations from the norm, their fragmentation and degeneration occur no earlier than in cases of pulp necrosis. Our results confirmed these statements, in the examined material we observed no changes concerning the nerve fibres with or without PGP9.5 expression. The richest innervation and vasculature of the pulp demonstrated by the expression of PGP9.5 as well as CD31 respectively, was found in pulp horns which confirms observations of other authors [21].

Our study showed that the malocclusion stress accompanying teeth crowding may result in the alterations of the normal structure of dental pulp in some apparently 'healthy' teeth. Future studies of the histological structure and function of the dental pulp of the teeth extracted for orthodontic reasons as well as the orthodontically-treated teeth can lead to improvements in clinical methods. This can be especially helpful in doubtful or problematic cases in 'border line' patients, when orthodontists have to make a decision about extraction treatment for teeth crowding. Our study suggests that dentists should not hesitate to extract teeth to obtain better orthodontic treatment results, since in many teeth which lack clinical signs of pulp disease pulp atrophy and focal inflammation can occur.

\section{References}

1. Pimenta FJGS, Sá AR, Gomez RS. Lymphangiogenesis in human dental pulp. Int Endod J. 2003;36:853-856.

2. Abi-Ramia LB, Stuani AS, Stuani AS, Stuani MB, Mendes Ade M. Effects of low-level laser therapy and orthodontic tooth movement on dental pulps in rats. Angle Orthod. 2010;80:116-122.

3. Gronthos S, Mankani M, Brahim J, Gehron Robey P, Shi S. Postnatal human dental pulp stem cells (DPSCs) in vitro and in vivo. Proc Natl Acad Sci U S A. 2000;97:13625-13630.

4. Rodd HD, Boissonade FM. Vascular status in human primary and permanent teeth and disease. Eur J Oral Sci. 2005;113:128-134.

5. Caviedes-Bucheli J, Lombana N, Azuero-Holguin MM, Munoz HR. Quantification of neuropeptides (calcitonin gene-related peptide, substance $P$, neurokinin A, neuropeptide $\mathrm{Y}$ and vasoactive intestinal polypeptide) expressed in healthy and inflamed human dental pulp. Int Endod J. 2006;39:394-400.

6. Di Nardo Di Maio F, Lohinai Z et al. Nitric oxide synthase in healthy and inflamed human dental pulp. J Dent Res. 2004;83:312-316.

7. Tønder KJH. Vascular reactions in the dental pulp during inflammation. Acta Odontol Scand. 1983;41:247-256.

8. Izumi T, Kobayashi I, Okamura K, Sakai H. Immunohistochemical study on the immunocompetent cells of the pulp in human non-carious and carious teeth. Arch Oral Biol. 1995;40:609-614.

9. Veberiene R, Smailiene D, Baseviciene N, Toleikis A, Machiulskiene $\mathrm{V}$. Change in dental pulp parameters in response to different modes of orthodontic force application. Angle Orthod. 2010;80:1018-1022.

10. Kayhan F, Küçükkeleş N, Demirel D. A histologic and histomorphometric evaluation of pulpal reactions following rapid palatal expansion. Am J Orthod Dentofacial Orthop. 2000;117:465-473.

11. Al Maaitah E, El Said N, Abu Alhaija ES. First premolar extraction effects on upper airway dimension in bimaxillary proclination patients. Angle Orthod. 2012; 82:853-859.

12. Tarazona B, Paredes V, Llamas JM, Cibrián R, Gandia J-L. Influence of first and second premolar extraction or non-extraction treatments on mandibular third molar angulation and position. A comparative study. Med Oral Patol Oral Cir Bucal. 2010;15:760-766.

13. Pulver WH, Taubman MA, Smith DJ. Immune components in normal and inflamed human dental pulp. Arch Oral Biol. 1977;22:103-111.

14. Freitas P, Novaretti CP, Rodini CO, Batista AC, Lara VS. Mast cells and lymphocyte subsets in pulps from healthy and carious human teeth. Oral Surg Oral Med Oral Pathol Oral Radiol Endod. 2007;103:95-102.

15. Gvozdenovic-Sedlecki S, Quist V, Hansen HP. Histologic variations in the pulp of intact premolars from young individuals. Scand J Dent Res. 1973;81:433-440. 
16. Digka A, Lyroudia K, Jirasek T, Kasampalidis IN, Karayannopoulou G, Kubinova L. Visualisation of human dental pulp vasculature by immunohistochemical and immunofluorescent detection of CD34: A comparative study. Aust Endod J. 2006;32:101-106.

17. Artese L, Rubini C, Ferrero G, Fiorini M, Santinelli A, Piattelli A. Vascular endothelial growth factor (VEGF) expression in healthy and inflamed human dental pulps.J Endod. 2002;28:20-23.

18. Sullivan CA, Ghosh S, Ocal IT, Camp RL, Rimm DL, Chung GG. Microvessel area using automated image analysis is reproducible and is associated with prognosis in breast cancer. Hum Pathol. 2009;40:156-165.
19. Santamaria MJr, Milagres D, Stuani AS, Stuani MB, Ruellas AC. Initial changes in pulpal microvasculature during orthodontic tooth movement: a stereological study. Eur J Orthod. 2006;28:217-220.

20. Zuza EP, Carrareto AL, Lia RC, Pires JR, de Toledo BE. Histopathological features of dental pulp in teeth with different levels of chronic periodontitis severity. ISRN Dent. 2012;2012:271350.

21. Alvarado LT, Perry GM, Hargreaves KM, Henry MA. TRPM8 axonal expression is decreased in painful human teeth with irreversible pulpitis and cold hyperalgesia. J Endod. 2007;33:1167-1171.

Submitted: 9 February, 2012 Accepted after reviews: 21 June, 2013 